\title{
Micodiversidade no Cerrado: relatos nos últimos anos
}

\author{
Héberly Fernandes Braga ${ }^{a *}$, Heloiza Ferreira Alves do Prado ${ }^{a} \bullet$ \\ a Universidade Estadual Paulista "Júlio de Mesquita Filho", Brasil \\ *Autor correspondente (heberly@iftm.edu.br)
}

\section{N F O}

\section{Keywords}

fungi of the cerrado bioprospecting

new genera

species

\begin{abstract}
A B S T R A C T
Mycodiversity in the Cerrado: reports in recent years.

The Cerrado is home to a huge variety of organisms, including the microbiota, which is still little known by science. Scoring scientific evidence that portrays the richness and abundance of microorganisms in this biome is important not only to stimulate more bioprospective research that demonstrates the biological potentialities present in the region, but also conservation and sustainable attitudes. This review aimed to highlight reports of new fungal species recently discovered in the Cerrado biome. An exploratory research was carried out with a survey of articles in English and Portuguese published between the years 2015 to 2019, in the Google Scholar, Scielo, Portal Capes and DOAJ databases, using the descriptors "fungi do cerrado", "(bio) prospecção of fungi from the cerrado "and" new species of fungi in the cerrado ". In the search, only articles that reported discoveries of new species for the scientific community or first report of a certain species in the Cerrado biome were considered, as well as the first evidenced association of a certain fungus with other organisms, and such reports may or may not indicate possible biotechnological applications of the organisms. found. Articles that did not meet the inclusion criteria were excluded from the search. Research was verified with reports of new discoveries, especially in the states of Goiás, Minas Gerais, Mato Grosso and Mato Grosso do Sul. Some of the discoveries showed possible biotechnological properties of the isolates found, emphasizing the need for actions aimed not only at the conservation of the biome, as well as more bioprospective research.
\end{abstract}

\section{R E S U M O}

O Cerrado alberga uma enorme variedade de organismos, incluindo a microbiota, que ainda é pouco conhecida pela ciência. Pontuar evidências ciêntíficas que retratem a riqueza e abundância dos microrganismos existentes nesse bioma é importante não somente para estimular mais pesquisas bioprospectivas que demonstrem as pontecialidades biológicas presentes na região, mas também atitudes conservacionistas e sustentáveis. A presente revisão teve por objetivo destacar relatos de novas espécies fúngicas descobertas recentemente no bioma Cerrado. Foi realizada pesquisa exploratória com o levantamento de artigos em inglês e português publicados entre nos anos de 2015 a 2019, nas bases Google Acadêmico, Scielo, Portal Capes e DOAJ, empregando-se os descritores "fungos do cerrado", "(bio)prospecção de fungos do cerrado" e "novas espécies de fungos no cerrado". Na busca foram considerados somente artigos que relatassem descobertas de novas espécies para a comunidade científica ou primeiro relato de uma determinada espécie no bioma Cerrado, bem como primeira associação evidenciada de determinado fungo a outros organismos, podendo tais relatos indicarem ou não possíveis aplicações biotecnológicas dos organismos encontrados. Artigos não enquadrados nos critérios de inclusão foram excluídos da busca. Foram verificadas pesquisas com relatos de novas descobertas em especial nos estados de Goiás, Minas Gerais, Mato Grosso e Mato Grosso do Sul. Algumas das descobertas evidenciaram possíveis propriedades biotecnológicas dos isolados encontrados, envidenciando a necessidade de ações que visem não somente a conservação do bioma, como também mais pesquisas bioprospectivas. 


\section{INTRODUÇÃO}

É sabido que o Cerrado é uma região biogeográfica reconhecida pela comunidade científica juntamente a outros habitats naturais como hotsopts ecológicos, isto é, ambientes que apresentam uma enorme quantidade e variedade de organismos, especialmente endêmicos, mas ameaçados de destruição. A alta biodiversidade dessa formação savânica não se resume somente à riqueza e abundância de espécies vegetais e animais, mas também a diversidade da microbiota, da qual muito pouco ainda se conhece.

O Cerrado é considerado o maior hotspot do hemisfério ocidental, e a savana tropical mais biodiversa do mundo (Sawyer et al., 2016), com uma extensão aproximada de 2 milhões de $\mathrm{km}^{2}$, sendo que cerca de $99,3 \%$ de sua área está no Brasil e o restante em partes dos territórios da Bolívia e Paraguai (Figura 01). Nele também estão presentes as três maiores nascentes de bacias hidrográficas da América do Sul: Amazonas/Tocantins, São Francisco e Prata.

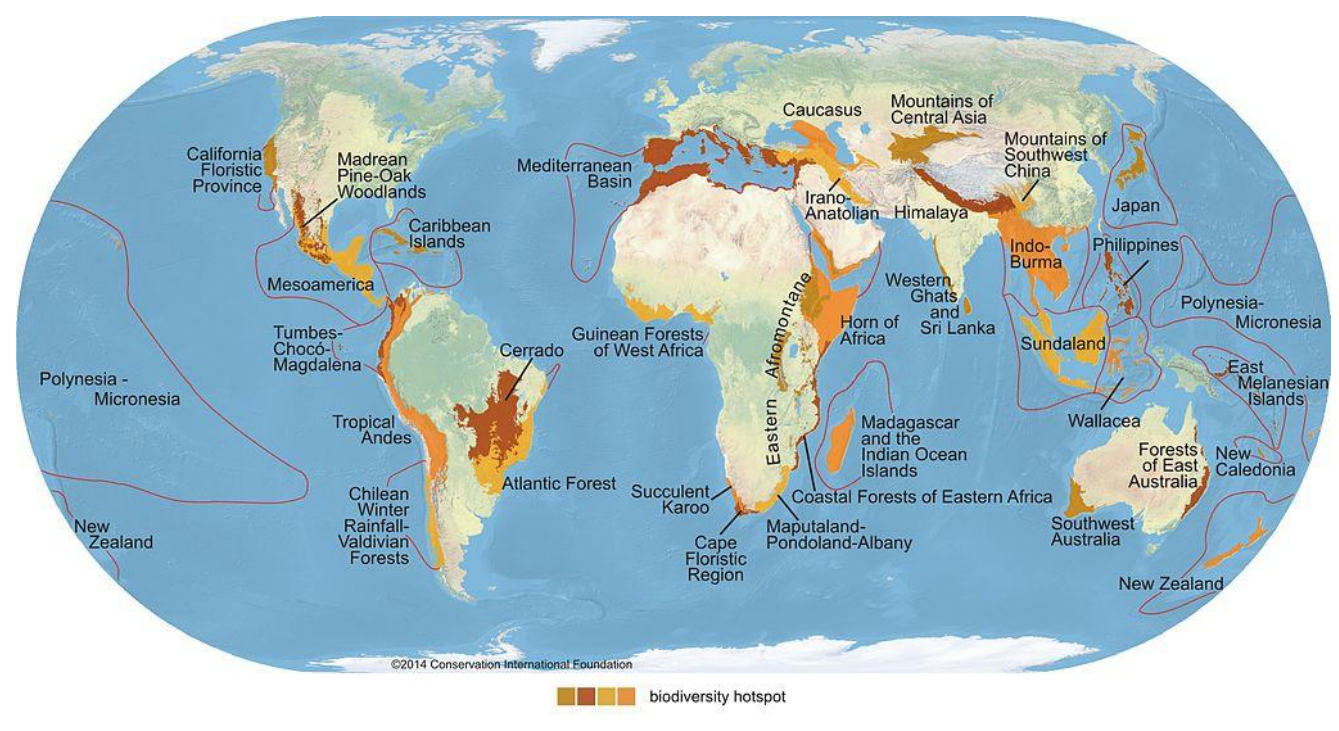

Conservation International (conservation.org) defines 35 biodiversity hotspots - extraordinary places that harbor vast numbers of plant and animal species found nowhere else. All are heavily threatened by habitat loss and degradation, making their conservation crucial to protecting nature for the benefit of all life on Earth.

Figura 1 - Mapa dos hotsposts de biodiversidade da Terra, incluindo o Cerrado.

Fonte: Conservation International (2014).

Essa alta biodiversidade do bioma, consequente de inúmeros processos evolutivos pelo qual o mesmo passou e tem passado, propicia a existência de organismos com distintas capacidades biológicas que permitem em primeira instância sua adaptação ao ambiente, mas que podem ser aproveitadas como produtos, processos ou serviços pelos seres humanos. Dessa forma, de acordo Souza et al. (2017), estudos que visem bioprospectar tais agentes são particularmente importantes.

Os fungos são organismos ubíquos reconhecidos como agentes que desempenham importante papel na decomposição e ciclagem de nutrientes. Além disso, muitas espécies interagem positivamente com outros seres por meio de relações mutualíticas essenciais. Outras são patógenos de plantas, animais e humanos. Dependendo das diferentes relações estabelecidas esses microrganimos demonstram potencial para serem empregados no controle biológico de pragas na agropecuária; podendo ainda atuar na produção biotecnológica de distintos produtos; e otimizar processos, melhorando a qualidade de determinados serviços e atividades humanas (Abreu et al., 2015).

Assim, a presente revisão teve como objetivo destacar relatos de pesquisas sobre novas espécies fúngicas descobertas recentemente no bioma Cerrado.

\section{MATERIAL E MÉTODOS}

Foi realizada pesquisa exploratória qualitativa nas bases de dados Google Acadêmico, Scielo, Portal Capes e DOAJ, considerando-se trabalhos publicados entre 2015 a 2019.

Como critérios de inclusão foram considerados artigos em inglês e português que somente relataram descobertas de novas espécies para a comunidade científica ou primeiro relato de uma determinada espécie no bioma Cerrado, bem como primeira associação evidenciada de determinado fungo a outros organismos, podendo tais relatos indicarem ou não possíveis aplicações biotecnológicas dos organismos encontrados.

Os seguintes descritores foram empregados na busca: "fungos do cerrado", "(bio)prospecção de 
fungos do cerrado" e "novas espécies de fungos no cerrado".

Artigos não enquadrados nos critérios de inclusão, assim como aqueles que somente relatassem quantificação da micobiota local; influências de diferentes manejos sobre a carga fúngica; variações da carga microbiana conforme diferentes tipos fitofisionômicos do Cerrado; aplicações de fungos já descritos e relatados anteriormente, ou espécies já descobertas e reclassificadas, foram excluídos da busca.

\section{RELATOS DE DESCOBERTAS RECENTES DE FUNGOS NO CERRADO}

A diversidade fúngica brasileira, até meados de 2010, estava descrita em poucas listas de espécies e em algumas publicações taxonômicas e ecológicas esparsas. Nos últimos anos, por meio da classificação pautada na análise filogenética e com a publicação on-line do Catálogo de Plantas e Fungos do Brasil, tem sido possível agregar o conhecimento disperso, acelerarando a descoberta de novos gêneros e espécies. $\mathrm{O}$ incremento de novas espécies catalogadas no Cerrado cresceu 45,6\% de 2010 para 2015 (Maia, et al. 2015).

Em pesquisa realizada por Castro (2015), em Brasília, visando determinar a micodiversidade associada a ramos e folhas de Copaifera langsdorffii (copaíba), uma planta leguminosa que apresenta distribuição concentrada nos biomas Amazônico e do Cerrado, sete fungos (de um total de oito descritos e depositados na Coleção Micológica do Herbário UB da Universidade de Brasília) pertencentes ao grupo dos ascomicetos e celomicetos foram relatados pela primeira vez para a hospedeira em questão. Desses, cinco (Stigmopeltis sp., Geastrumia sp., Metathyriella sp., Treubiomyces sp. e Leptosphaeria sp.) são prováveis espécies novas para a ciência e dois são possíveis novos gêneros, segundo o autor.

Lopes et al. (2016), utilizaram bagaço de cana proveniente de usina de álcool e açúcar localizada na região centro-sul do Cerrado (Goiás - GO), na tentativa de se isolar e identificar linhagens fúngicas com potencial aplicação na indústria do bioetanol. Empregando identificação fenotípica clássica e métodos moleculares, os autores identificaram 20 espécies, sendo três delas pertencentes ao gênero Paecilomyces: $P$. formosus, $P$. saturatus e $P$. parvisporus. Foi verificado, por meio de consulta à base de dados speciesLink (http://www.splink.org.br), que $P$. parvisporus e $P$. formosus, isolados e identificados no trabalho, foram os primeiros relatos científicos formais de ocorrência das espécies em território brasileiro. Além disso, a ocorrência de $P$. saturatus é citada pela primeira vez dentro do domínio do cerrado (Figura 02). Ressalta-se que a presença de Paecilomyces sp. em fontes lignocelulósicas evidencia a capacidade bioconversora desses fungos frente a açúcares complexos como a celulose (Basso et al., 2010).

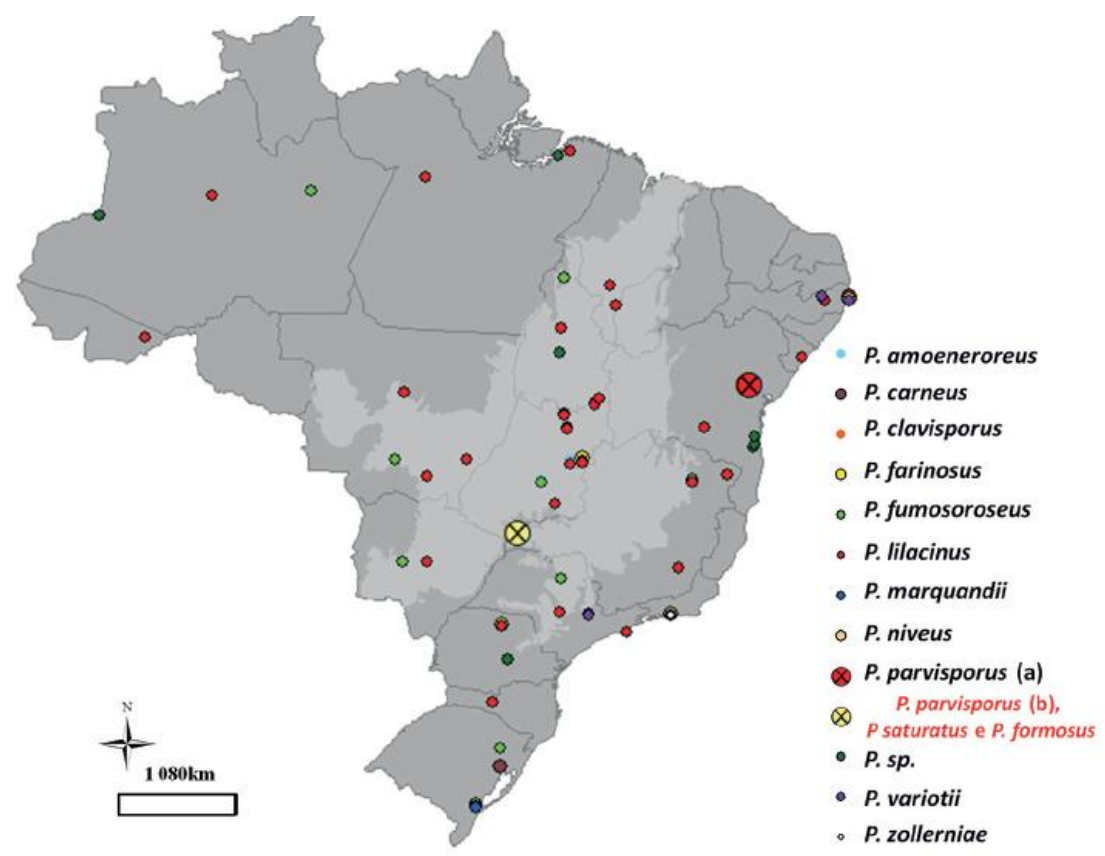

Fonte: Lopes et al. (2016).

Figura 2 - Distribuição geográfica de espécies de Paecilomyces no Brasil, inferida a partir de dados recuperados da base speciesLink. O círculo vermelho destaca a ocorrência de Paecilomyces parvisporus Y. F. Han \& Z. Q. Liang na Bahia e o círculo amarelo refere-se às coordenadas de amostragem das espécies identificadas. 
Outro estudo exploratório feito no Cerrado, ao longo de três décadas, visando identificar fungos micorrízicos arbusculares (FMA) do filo Glomeromycota, encontrou 92 espécies, das quais sete (Acaulospora reducta, Ambispora brasilienses, Cetraspora auronigra, Dentiscutata cerradensis, D. scutata, Paraglomus brasilianum e Racocetra verrucosa) apresetaram o primeiro registro para a área savânica, sendo A. brasilienses e $C$. auronigra, reportadas exclusivamente na região fitofisionônica campos rupestres (Jobim et al., 2016). Pesquisa realizada em Diamantina (Minas Gerais - MG) visando avaliar a ocorrência de FMA associados a raízes de Syngonanthus elegans, uma planta encontrada em formações rupestres do cerrado e em vias de extinção, também sugere novas espécies de fungos desconhecidos pela ciência (Costa et al., 2016).

Teixeira et al. (2017), avaliando a diversidade de esporos de FMA em solo da área de mineração e do entorno da região do Quadrilátero Ferrífero (MG), relataram a ocorrência pela primeira vez no Brasil das espécies Acaulospora nivalis e A. alpina. Os autores ainda evidenciaram que ambientes do Quadrilátero Ferrírero são hotspots de diversidade de FMA, evidenciando a necessidade de pesquisas prospectivas mais aprofudadas e de uma melhor conservação dessa região.

Noriler et al. (2018), realizaram coletas entre janeiro a junho de 2016 em folhas de Stryphnodendron adstringens (popularmente conhecido como barbatimão), de ocorrência no Cerrado, visando selecionar fungos endofíticos. Por meio de identificação pelo sequenciamento do espaçador interno transcrito (ITS) da região do rDNA, os autores sugeriram possíveis novas espécies (Diaporthe sp. e Phaeophleospora sp.). Os pesquisadores ainda testaram a atividade antimicrobiana dos isolados encontrados contra patógenos agrícolas e clínicos e foi verificado que alguns dos isolados de Diaporthe sp. inibiu mais que $60 \%$ do crescimento micelial Phyllosticta citricarpa e Colletotrichum abscissum, fungos estes causadores de muitos problemas na citricultura; e moderada $(++)$ a alta $(+++)$ atividade com amplo espectro contra Staphylococcus aureus meticilinoresistentes (MRSA), Pseudomonas aeruginosa,
Acinetobacter baumannii e Klebsiella pneumoniae produtora de carbapenemase (KPC). Um dos isolados de Phaeophleospora sp. também apresentou alta atividade contra $S$. aureus (MRSA).

Coletas realizadas por Alvarenga et al. (2015), ao longo de 14 anos (2001 a 2014), em diferentes áreas do estado de Goiás, reportaram e ampliaram o conhecimento da ocorrência do basiomiceto Auricularia no Brasil. Das 101 amostras examinadas, $54 \%$ foram representadas pela espécie A. nigricans, $20 \%$ por $A$. fuscosuccinea, $13 \%$ por A. mesenterica e $12 \%$ por $A$. delicata (Figura 03). Conforme os autores, esses são os primeiros registros de A. fuscosuccinea, A. mesenterica e A. delicata para o estado de Goiás e de A. delicata para o Distrito Federal.

Uma bioprospecção de fungos coprófilos realizada em Goiás na unidade de conservação Parque da Serra de Caldas Novas, registrou uma nova variedade do fungo ascomiceto Delitschia gigaspora, denominada pescanii, em esterco de vaca (Calaça et al., 2015). Outro estudo publicado em 2016 pelo mesmo autores, registrou novas espécies de fungos desse mesmo grupo em estercos de cavalo (Equus caballus L.), bovinos (Bos taurus L.), queixada (Tyassu pecari Link) e porco doméstico (Sus domesticus L.) coletados também em diferentes localidades do estado de Goiás, dentro do domínio morfoclimático do Cerrado (Figura 04). Foram identificadas as seguintes espécies: Pyxidiophora arvernensis, Zopfiella latipes e Hypocopra stercoraria (primeiros registros dos gêneros no Brasil e primeiras espécies na região Neotropical); Cercophora coronata (primeiro registro da espécie para região centrooeste do Cerrado e segundo para o Brasil) (Figura 05).

É importante ressaltar que os fungos coprófilos são organismos especializados em se desenvolver e assimilar nutrientes não absorvidos, e presentes em esterco de animais, tendo um papel primordial na bioconversão e recligagem desses rejeitos no ambiente. Embora sejam cosmopolitas são mais frequentemente registrados na Europa, América do Norte e alguns países neotropicais (Doveri, 2004; Masunga et al., 2006). 

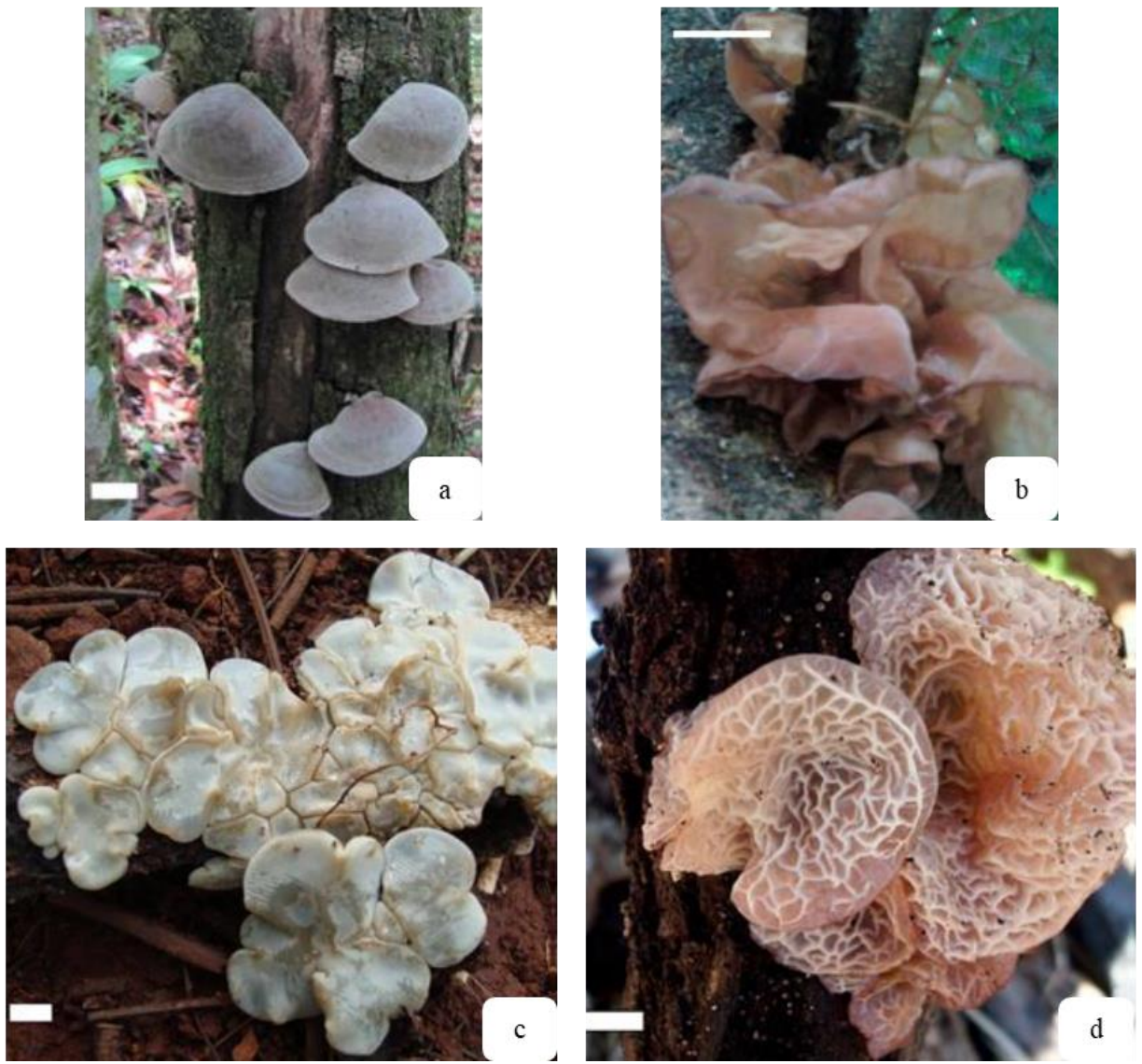

Figura 3 - Basidiomas de A. nigricans (a), A. fuscosuccinea (b), A. mesentérica (c) e A. delicata (d). Fonte: Avarenga et al. (2015). Barras de escala: a) $3 \mathrm{~cm}$; b) $1 \mathrm{~cm}$; c) $3 \mathrm{~cm}$; d) $2 \mathrm{~cm}$.

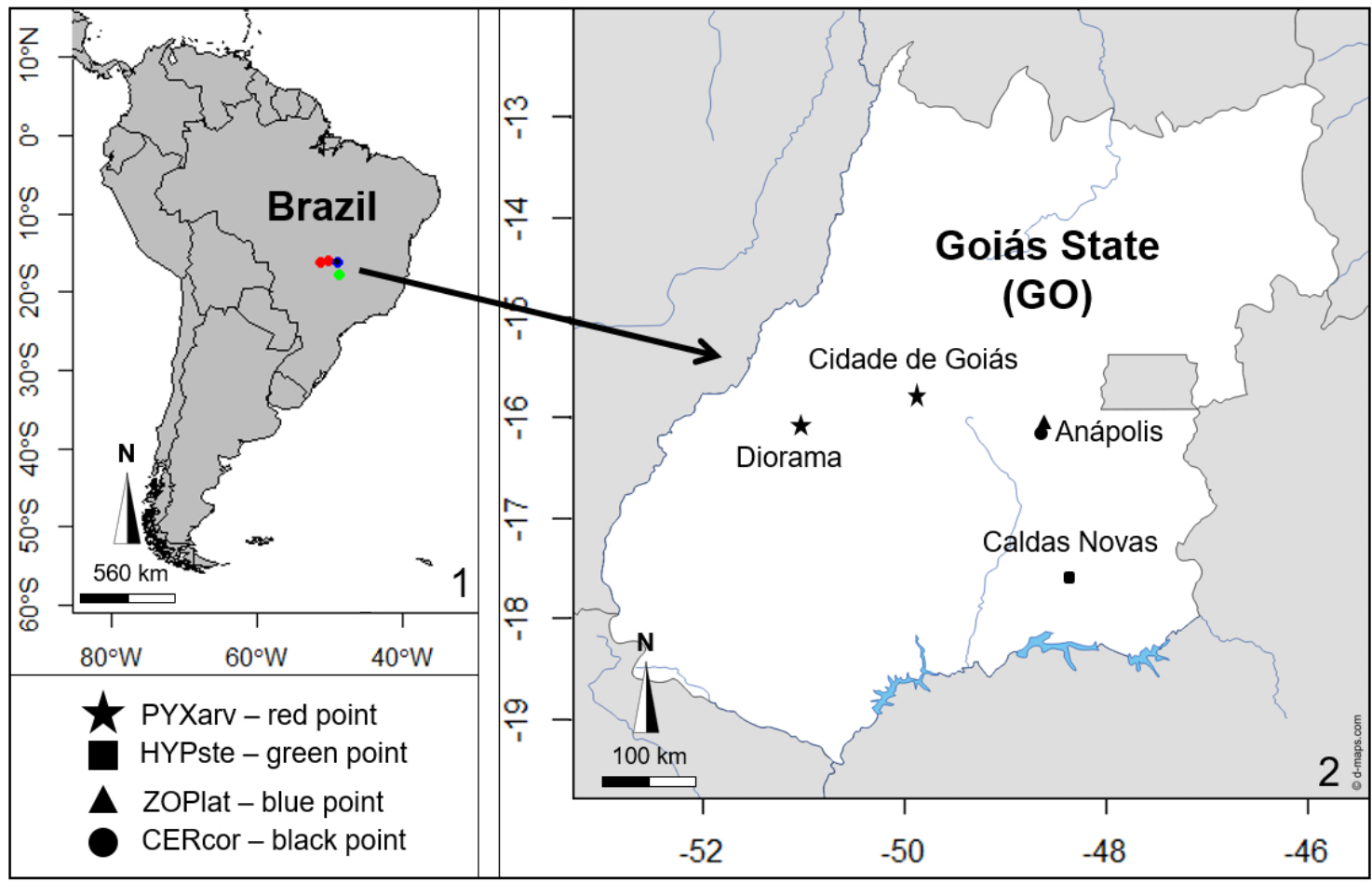

Figura 4 - Mapa das áreas de localização de novos registros de fungos coprófilos descobertos no Brasil (a), e área de coleta dos estercos animais (b). PYXarv: Pyxidiophora arvernensis; HYPste: Hypocopra stercoraria; ZOPlat: Zopfiella latipes; CERcor: Cercophora coronata. Fonte: Calaça \& Xavier-Santos (2016). 

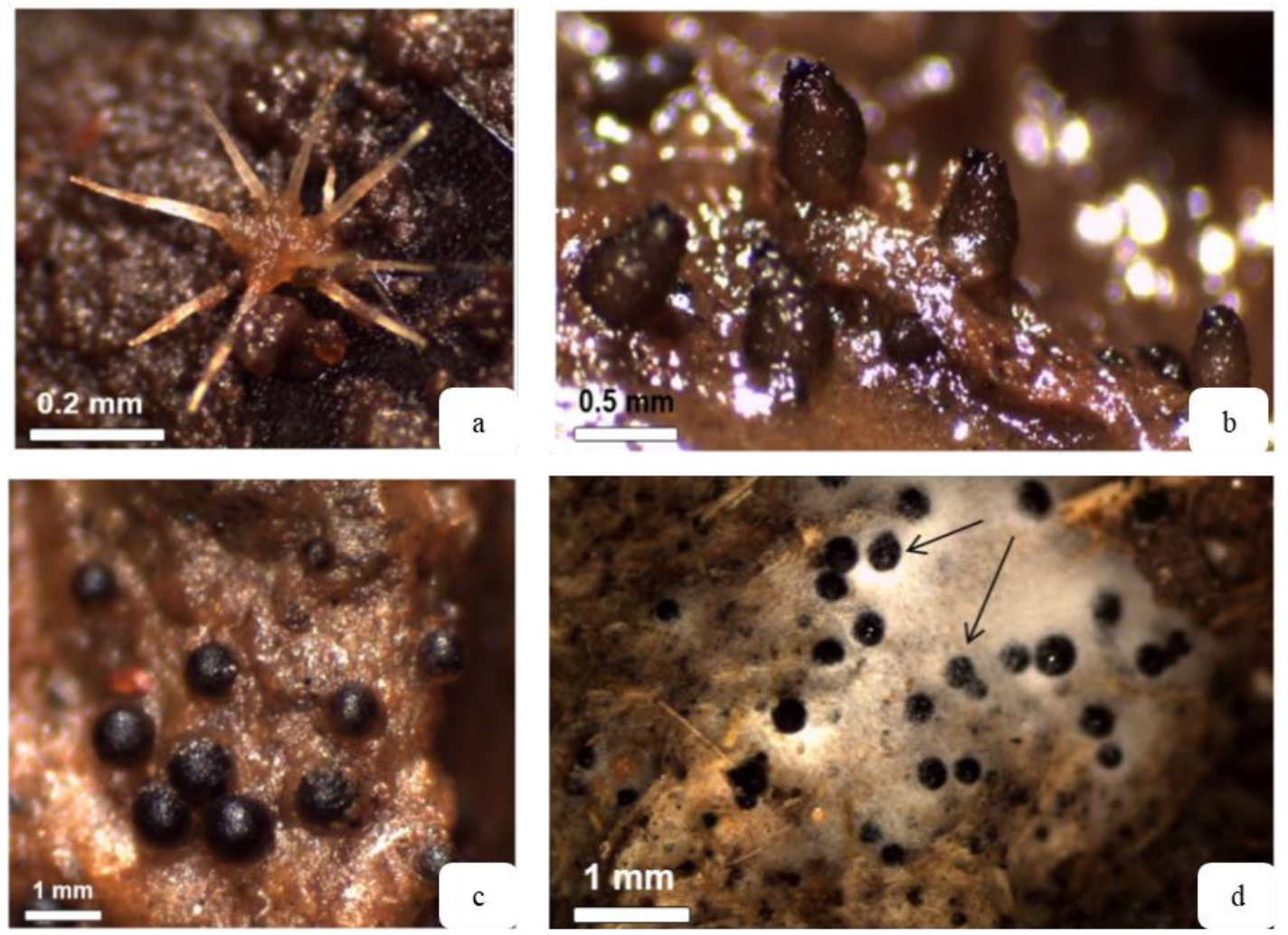

Figura 5 - Peritécios de Pyxidiophora arvernensis (a); Cercophora coronata (b); Hypocopra stercoraria (d); e cleitotécios de Zopfiella latipes (c) sobre esterco animal.

Fonte: Calaça \& Xavier-Santos (2016).
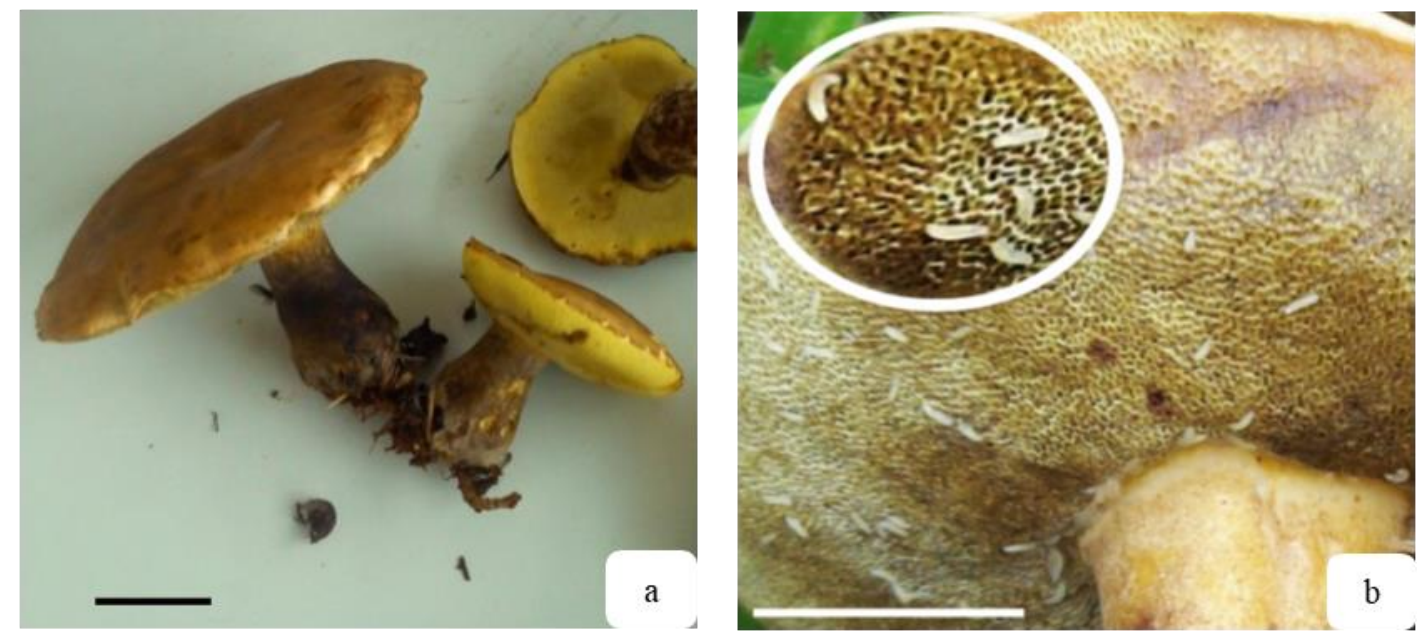

Figura 6 - Basidioma fresco de P. beniensis (a) e detalhe da micofagia por larvas de inseto (b).

Fonte: Calaça et al. (2018). Barras de escala: a) $2 \mathrm{~cm}$; b) $2 \mathrm{~cm}$.

Em outro estudo também realizado em regiões de cerrado do estado de Goiás, entre 2008 a 2014, Alvarenga e Xavier-Santos (2017) citaram a ocorrência de basiomicetos gelatinosos que possuem atividade celulásica semelhante a fungos altamente hidrolíticos pertencentes a antiga ordem Aphyllophorales. Dentre eles, foi identificado Calocera cornea como primeira e única citação em
40 anos para a capital brasileira e segundo registro para o bioma Cerrado. Além disso, Dacrymyces chrysospermus, Dacryopinax elegans, D. maxidorii, D. spathularia foram identificados como primeiras citações para o Cerrado e região Centro-Oeste do Brasil.

Calaça et al. (2018), em visitas aos munícipios de Anápolis (GO) e Rio Quente (GO) durante 
período chuvoso, registraram pela primeira vez no Cerrado o fungo basidiomiceto Phlebopus beniensis (Figura 06a). Os autores ainda relataram a micofagia de larvas de insetos sobre o fungo (Figura 06b), algo pouco conhecido no Brasil e com alguns registros esparsos. Segundo Yamashita et al. (2015), a fonte nutritiva ofertada pelos fungos pode contribuir para estruturar a redes alimentares das comunidades de insetos, tornando-se componentes chave para a manutenção dos ecossistemas.

Alguns levantamentos bibliográficos tem mostrado que ainda são poucas as descobertas relativas à enorme diversidade fúngica existente no Cerrado. Rivas e Niveiro (2019) realizaram um levantamento de fungos agaricoides citados no Paraguai entre 1883 a 2017 por meio de checklist nos bancos de dados Mycobank (www.mycobank.org) e Index Fungorum (www.indexfungorum.org) e verificaram poucos registros de identificação de espécies nas regiões biogeográficas de Chaco, Pantanal e Cerrado, demonstrando ainda a baixa exploração científica de tais fungos nessas respectivas áreas. Um levantamento bibliográfico realizado por Alvarenga e Xavier-Santos (2015) sobre a distribuição de fungos gelatinosos (Agaricomycotina: Basidiomycota) nos estados brasileiros, também corrobora a necessidade de estudos prospectivos e taxonômicos sobre o grupo, haja visto que em $40 \%$ dos estados da federação não há ocorrência e a maioria das espécies é representada por um único registro.

Em coletas realizadas por Bononi et al. (2017) na região central do estado de Mato Grosso do Sul (MS), centro-oeste do Brasil, foram identificadas 18 espécies de fungos basidiomicetos: Conocybe crispa, Gymnopus dryophilus, Marasmius androsaceus, Marasmius oreades, Mycena pura, Rhodocollybia butyraceae, Pleurotus eryngii, Coprinellus micaceus, Panaeolus rickenii, Hypholoma fasciculare, Clitocybe infundibuliformis, Tricholoma argyraceum, Fomitiporia robusta, Cymatoderma elegans, Athelopsis galzinii, Trametes ellipsospora, Guepinia helvelloides, Tremella mesentérica (Figura 7) ainda não citadas e listadas nacionalmente e 36 espécies com primeira citação para o Estado. Do total de 62 espécies de basidiomicetos coletados, os autores identificaram 15 comestíveis, quatro tóxicas, 10 com propriedades medicinais, duas com potencialidade de aplicação em processos de biorremediação e uma com propriedade bioluminescente, conforme comparações à literatura disponível.
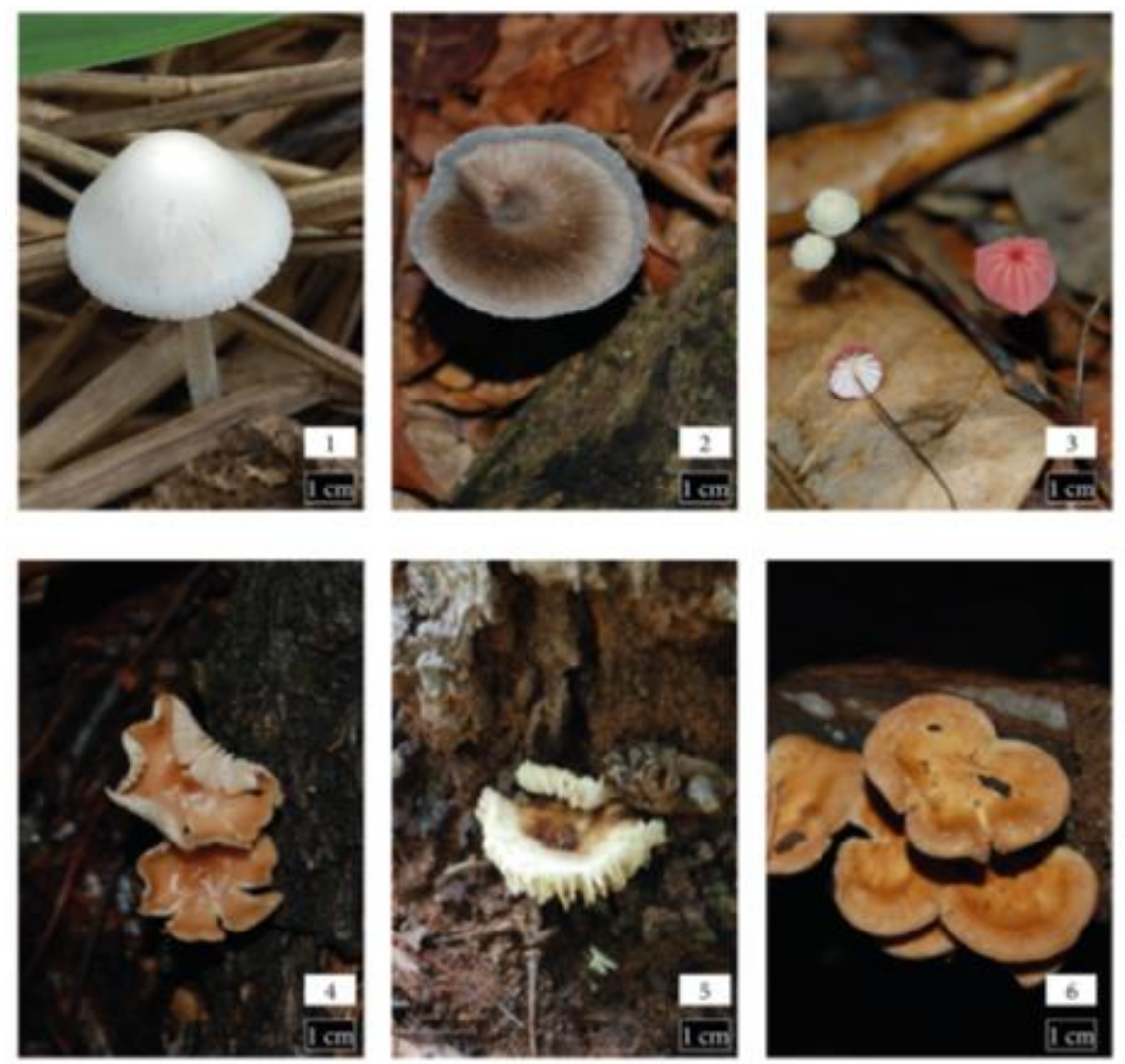

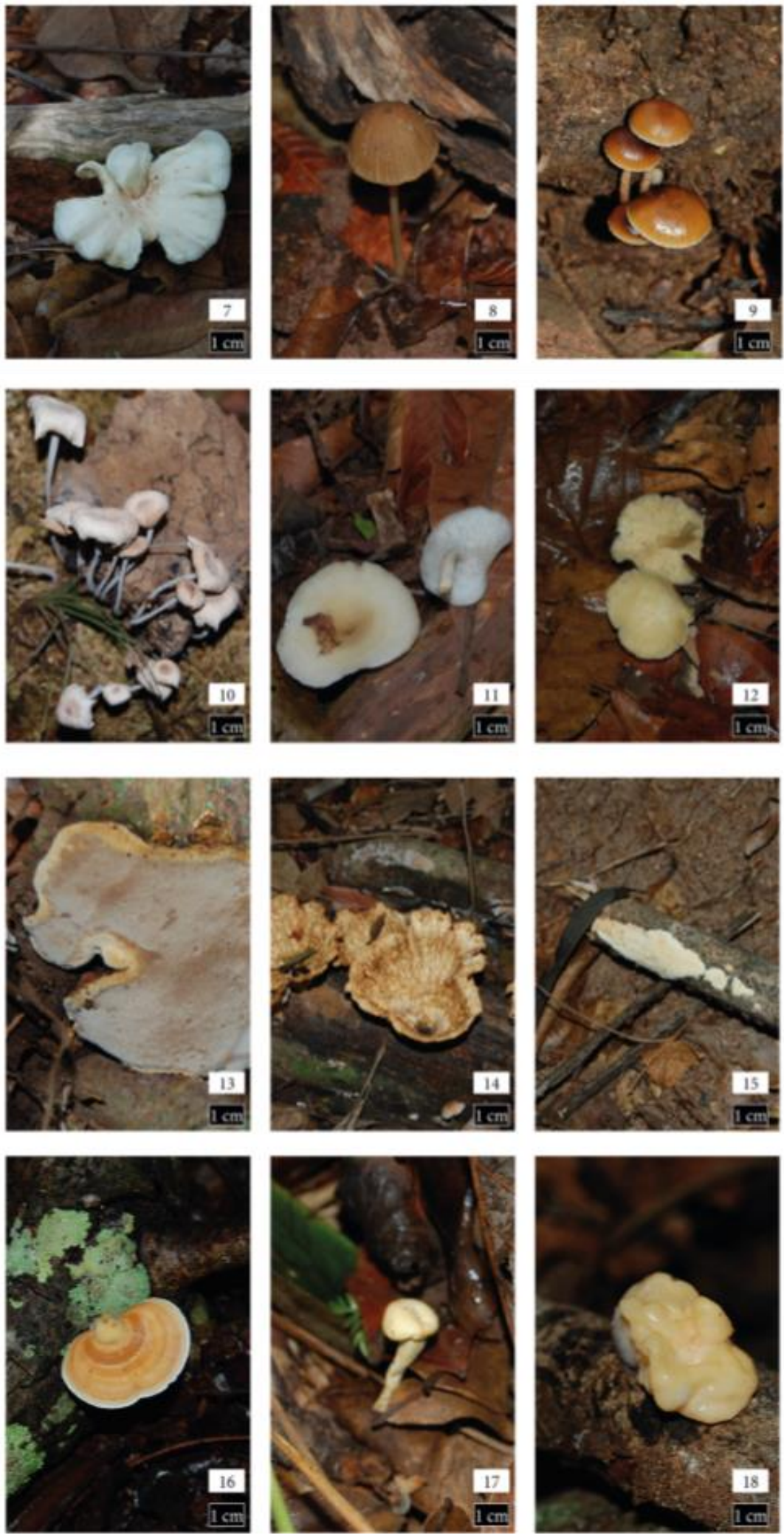

Fonte: Bononi et al. (2017).

Figura 7 - Agaricomicetos da Serra de Maracaju, Mato Grosso do Sul, Brasil. Conocybe crispa (1). Gymnopus dryophilus (2). Marasmius androsaceus (3). Marasmius oreades (4). Mycena pura (5). Rhodocollybia butyraceae (6). Pleurotus eryngii (7). Coprinellus micaceus (8). Panaeolus rickenii (9). Hypholoma fasciculare (10). Clitocybe infundibuliformis (11). Tricholoma argyraceum (12). Fomitiporia robusta (13). Cymatoderma elegans (14). Athelopsis galzinii (15). Trametes ellipsospora (16). Guepinia helvelloides (17). Tremella mesenterica (18). 
Conforme Costa-Rezende et al. (2015), o conhecimento sobre a diversidade de fungos associados a madeira podre é ainda muito pouco explorado, especialmente no ambiente do Cerrado. Nesse sentido, em expedições realizadas no Parque Nacional da Chapada dos Guimarães, em Mato Grosso (MT), os pesquisadores identificaram cinco novas espécies como primeiros registros para o Cerrado: Hornodermoporus martius, Perenniporia aurantiaca, P. tephropora, Pyrofomes lateritius, Truncospora detrita.

\section{CONCLUSÕES}

As descobertas recentes elencadas demonstram a importância do Cerrado como um bioma altamente biodiverso, em especial no que tange a micobiota, e corrobora a necessidade de ações urgentes que visem não somente sua conservação, mas também de pesquisas visando bioprospectar novas espécies de microrganismos e/ou seus possíveis metabólitos que podem vir a serem empregados na melhoria de processos, produtos ou serviços ao homem.

\section{AGRADECIMENTOS}

Ao Instituto Federal de Educação, Ciência e Tecnologia do Triângulo Mineiro (IFTM), Campus Uberlândia Centro por possibilitar a oportunidade de realização da pesquisa em dedicação integral.

\section{REFERÊNCIAS BIBLIOGRÁFICAS}

Abreu JAS, Rovida AFS, Pamphile JA. Fungos de interesse: aplicações biotecnológicas. Revista Uningá Review, v.21, n. 1, p. 55-59, 2015.

Alvarenga RLM, Naves LRR, Xavier-Santos S. The genus Auricularia Bull. ex Juss. (Basidiomycota) in Cerrado (brazilian savanna) areas of Goiás state and the Federal District, Brazil. Mycosphere, v. 6, n. 5, p. 532-541, 2015. https://dx.doi.org/10.5943/mycosphere/6/5/3

Alvarenga RLM, Xavier-Santos S. A checklist of jelly fungi (Agaricomycotina: Basidiomycota) recorded in Brazil. Mycotaxon, v. 130, n. 3, p. 925-926, 2015. https://doi.org/10.5248/130.925

Alvarenga RLM, Xavier-Santos S. New records of Dacrymycetes (Fungi: Basidiomycota) from the Cerrado biome (brazilian savanna) and Midwest region, Brazil. Checklist, v. 13, n. 4, p. 335-342, 2017. https://doi.org/10.15560/13.4.335

Basso TP, Gallo CR, Basso LC. Atividade celulolítica de fungos isolados de bagaço de cana-de-açúcar e madeira em decomposição. Pesquisa Agropecuária Brasileira, v. 45, n. 11, p.1282-1289, 2010. http://dx.doi.org/10.1590/S0100204X2010001100008

Bononi VLR, Oliveira AKM, Gugliotta AM, Quevedo JR. Agaricomycetes (Basidiomycota, Fungi) diversity in a protected area in the Maracaju Mountains, in the brazilian central region. Hoehnea, v. 44, n. 3, p. 361-377, 2017. http://dx.doi.org/10.1590/2236-8906-70/2016

Calaça FJS, Delpont M, Xavier-Santos S. Delitschia gigaspora var. pescanii: a new variety of coprophilous fungus from Brazil. Mycosphere, v. 6, n. 1, p. 122-126, 2015. http://dx.doi.org/10.5943/mycosphere/6/1/12

Calaça FJS, Magnago AC, Alvarenga RLM, Xavier-Santos S. Phlebopus beniensis (Boletinellaceae, Boletales) in the brazilian Cerrado biome. Rodriguésia, v. 69, n. 2, p. 939944, 2018. http://dx.doi.org/10.1590/2175$786020186924 \times 6$

Calaça FJS, Xavier-Santos S. New records of coprophilous ascomycetes (Fungi: Ascomycota) from Brazil and Neotropical Region. Check List, v. 12, n. 6, p.1-9, 2016. http://dx.doi.org/10.15560/12.6.2009

Castro MT. Micodiversidade associada a árvores de Copaifera langsdorffii Desf. em Brasília, Distrito Federal. Flor@m Floresta e Ambiente, v. 22, n. 2, p. 256-261, 2015. http://dx.doi.org/10.1590/2179-8087.094914

Conservation International. Biodiversity hotsopsts map. 2014. https://commons.wikimedia.org/wiki/File:Biodiversity_Hot spots_Map.jpg

Costa HAO, Stürmer SL, Ragonezi C, Grazziotti PH, Grazziotti DCFS, Silva EB. Species richness and root colonization of arbuscular mycorrhizal fungi in Syngonanthus elegans, an endemic and threatened species from the Cerrado domain in Brazil. Ciência e Agrotecnologia, v. 40, n. 3, p. 326-336, 2016. http://dx.doi.org/10.1590/1413-70542016403046815

Costa-Rezende DH, Ferreira-Lopes V, Salvador-Montoya CA, Alves-Silva G, Mello A, Drechsler-Santos ER. New records of Perenniporia sensu lato and Pyrofomes for de brazilian Cerrado. Iheringia Série Botânica, v. 70, n. 1, p. 157-166, 2015.

Doveri F. Fungi Fimicoli Italici: a guide to the recognition of Basidiomycetes and Ascomycetes living on faecal material. Bresadola: Scientific Committee of the Bresadola Mycological Association (A.M.B.), 1104p. 2004.

Jobim K, Oliveira BIS, Goto BT. Checklist of the Glomeromycota in the brazilian savana. Mycotaxon, v. 131, n. 1, p. 255-256, 2016. https://doi.org/10.5248/131.255

Lopes AM, Vitorino LC, Castro CFS, Nascimento JPL, Souchie EL. Primeiro relato da ocorrência de Paecilomyces formosus e Paecilomyces parvisporus no Brasil. Revista Brasileira de Biociências, v. 14, n. 4, p. 215-224, 2016.

Maia LC, Carvalho Júnior AA, Cavalcanti LH, Gugliotta AM, Drechsler-Santos ER, Santiago ALMA et al. Diversity of brazilian fungi. Rodriguésia, v. 66, n. 4, p. 1033-1045, 2015. http://dx.doi.org/10.1590/2175-7860201566407

Masunga GS, Andresen $\varnothing$, Taylor JE, Dhillion SS. Elephant dung decomposition and coprophilous fungi in two habitats of semi-arid Botswana. Mycological Research, v. 110, n. 10, p. 1214-1226, 2006 https://doi.org/10.1016/j.mycres.2006.07.004

Noriler AS, Savi DC, Aluizio R, Palácio-Cortes AM, Possiede YM, Glienke C. Bioprospecting and structure of fungal endophyte communities found in the brazilian biomes, Pantanal, and Cerrado. Frontiers in Microbiology, v. 9, e1526, 2018. https://doi.org/10.3389/fmicb.2018.01526 
Rivas AF, Niveiro N. Checklist of the agaricoid fungi from Paraguay. Mycotaxon, v. 133, n. 4, p. 729-729, 2019. https://doi.org/10.5248/133.729

Sawyer D, Mesquita B, Coutinho B, Almeida FV, Figueiredo I, Lamas I et al. Perfil do ecossistema hotspot de biodiversidade do Cerrado. Critical Ecosystem Partnership Fund, 520p. 2016. http://cepfcerrado.iieb.org.br/wpcontent/uploads/2017/09/PR_Cerrado-Perfil-do-

Ecossistema_TEXTOAPENDICES_port_revisada20170804.compressed.pdf

Souza JVB, Sousa DRT, Celestino JR, Segundo WOPF, Souza ÉS. Bioprospecting with brazilian fungi. In: Paterson R, Lima $N$ (Ed.). Bioprospecting: success, potential and constraints. 1 ed. v. 16. Cham: Springer, p.197-211. 2017.

Teixeira AFS, Kemmelmeier K, Marascalchi MN, Stürmer SL, Carneiro MAC, Moreira FMS. Arbuscular mycorrhizal fungal communities in an iron mining area and its surroundings: Inoculum potential, density, and diversity of spores related to soil properties. Ciência e Agrotecnologia, v. $41, \quad$ n. $5, \quad$ p. 511-525, 2017. http://dx.doi.org/10.1590/1413-70542017415014617

Yamashita S, Ando K, Hoshina H, Ito N, Katayama Y, Kawanabe $\mathrm{M}$ et al. Food web structure of the fungivorous insect community on bracket fungi in a Bornean tropical rain forest. Ecological Entomology, v. 40, n. 4, p. 390-400, 2015. https://doi.org/10.1111/een.12200 